\title{
Dark matter from SUGRA GUTs: mSUGRA, NUSUGRA and Yukawa-unified SUGRA
}

\author{
Howard Baer \\ Dep't of Physics and Astronomy, University of Oklahoma, Norman, OK 73019
}

\begin{abstract}
Gravity-mediated SUSY breaking models with $R$-parity conservation give rise to dark matter in the universe. I review neutralino dark matter in the minimal supergravity model (mSUGRA), models with non-universal soft SUSY breaking terms (NUSUGRA) which yield a well-tempered neutralino, and models with unified Yukawa couplings at the GUT scale (as may occur in an $S O(10)$ SUSY GUT theory). These latter models have difficulty accommodating neutralino dark matter, but work very well if the dark matter particles are axions and axinos.
\end{abstract}

Keywords: Supersymmetry, dark matter

PACS: $11.30 . \mathrm{Pb}, 12.60 . \mathrm{Jv}, 14.80 . \mathrm{Ly}$

This talk covers several highlights from research into supersymmetric dark matter by our research group over the past several years.

\section{NEUTRALINO DARK MATTER IN THE MSUGRA MODEL}

The minimal supergravity model[1], mSUGRA or CMSSM, is the paradigm model for many investigations of supersymmetry phenomenology. The mSUGRA model has the MSSM embedded in a supergravity framework, and then arranges for supergravity breaking via the super-Higgs mechanism, in a so-called hidden sector of the theory. Upon supergravity breaking, the gravitino acquires a mass of order $m_{3 / 2} \sim M^{2} / M_{P l} \sim 1$ $\mathrm{TeV}$, so that $M \sim 10^{11} \mathrm{GeV}$. Soft SUSY breaking (SSB) terms are induced due to the breakdown of supergravity, leading to weak scale SSB masses for gauginos, scalars, trilinear and bilinear soft terms. The defining assumption for mSUGRA is that at the GUT scale all scalars receive a common mass $m_{0}$, all gauginos receive a common mass $m_{1 / 2}, A$-terms receive a common mass $A_{0}$ and a bilinear mass term $B$ is also induced. The SSB terms evolve from $M_{G U T}$ down to $M_{\text {weak }}$ according to the renormalization group equations (RGEs). In particular, the up-Higgs soft mass $m_{H_{u}}^{2}$ is driven to negative values by the large top quark Yukawa coupling, causing a breakdown in electroweak symmetry (EWSB). The scalar potential minimization conditions allow one to trade the parameter $B$ for $\tan \beta$, the ratio of Higgs field vevs, while the magnitude (but not the sign) of the superpotential Higgs mass term $\mu$ is fixed in terms of the measured value of $M_{Z}$. The well-known parameter space

$$
m_{0}, m_{1 / 2}, A_{0}, \tan \beta, \operatorname{sign}(\mu)
$$

allows one to calculate all sparticle masses, mixings, scattering cross sections, decay rates and, in the case of the lightest neutralino $\widetilde{Z}_{1}$ (assumed to be the stable lightest 
SUSY particle (LSP), and a good WIMP dark matter candidate), the relic dark matter abundance[2].

The WMAP collaboration[3], and other groups, have measured the dark matter abundance of the universe to be $\Omega_{C D M} h^{2} \simeq 0.11$, which highly constrains models of new physics containing dark matter candidates, and in this case the mSUGRA model. In Fig. 1 the $m_{0} v s . m_{1 / 2}$ plane of mSUGRA parameter space is shown for $\tan \beta=50, A_{0}=0$ and $\mu<0$. We use isajet for our spartciel mass computations [4]. The red regions are not allowed due to (left-edge) the presence of a charged, stable stau $\tilde{\tau}_{1}$ LSP (in conflict with negative searches for charged/colored relic from the Big Bang), or lack of appropriate breakdown of electroweak symmetry (lower-right red region). The green-shaded regions give $\Omega_{\widetilde{Z}_{1}} h^{2} \leq 0.13$, and so are in accord with WMAP measurements; the white-shaded regions give $\Omega_{\widetilde{Z}_{1}} h^{2}>0.13$ and so are presumably excluded by the measured dark matter abundance.

The dark matter allowed regions consist of:

- A bulk region at low $m_{0}$ and low $m_{1 / 2}$ where neutralinos annihilate via $t$-channel slepton exchange (covered over here by red because we are at such high $\tan \beta$ ).

- The stau co-annihilation region where $\widetilde{Z}_{1}-\tilde{\tau}_{1}$ can co-annihilate due to their small mass gap (very thin sliver adjacent to red region at low $m_{0}$.

- The focus point (FP) region at large $m_{0}$ where $\mu$ becomes small and $\widetilde{Z}_{1}$ becomes mixed bino-higgsino dark matter.

- The $A$-annihilation funnel in the middle of the plot where $\widetilde{Z}_{1} \widetilde{Z}_{1}$ annihilation through the $A$-resonance is enhanced because $2 m_{\widetilde{Z}_{1}} \simeq m_{A}$.

- There is also a stop co-annihilation region for specific $A_{0}$ values, and a light Higgs $h$-resonance annihilation region possible at low $m_{1 / 2}$ for lower $\tan \beta$ values.

We also super-pose on the plot the approximate reach of the Fermilab Tevatron via clean trilepton channel for $10 \mathrm{fb}^{-1}$ of integrated luminosity, and the LHC reach for $100 \mathrm{fb}^{-1}$ of integrated luminosity. The LHC reach[5] covers essentially all the stau coannihilation region and most of the $A$-funnel, but a long strip of FP region extends away from the LHC reach where gluinos and squarks are very heavy, but charginos are quite light and higgsino-like, since $\mu$ is small. Notice in this region the reach of a linear $e^{+} e^{-}$ collider can exceed that of LHC, since chargino pair production is easy to see at linear colliders, but hard to see at LHC[6].

We also show contours of direct dark matter detection (DD), for experiments such as CDMS, Xenon-100, LUX or WARP (black contour). Note that this contour covers the FP region, so if SUSY lies in the FP region, with $m_{1 / 2} \gtrsim 700 \mathrm{GeV}$, then DD experiments will soon find a signal, while LHC may see none!

The magenta contour labelled $\mu$ denotes the approximate reach of the IceCube neutrino detector: it also covers most of the FP region. We also show approximate reach contours for indirect dark matter detection (IDD) via positrons, anti-protons and $\gamma$-rays arising from WIMP annihilation in the galactic halo[7]. These contours fill much of the $A$-funnel and also the FP region. In the case of the $A$-funnel, halo neutralinos have an enhanced annihilation rate through the $A$-resonance [8], while halo annihilations through $h$ and $H$ are suppressed, since $\sigma \cdot v \rightarrow 0$ as the WIMP velocity $v \rightarrow 0$. 


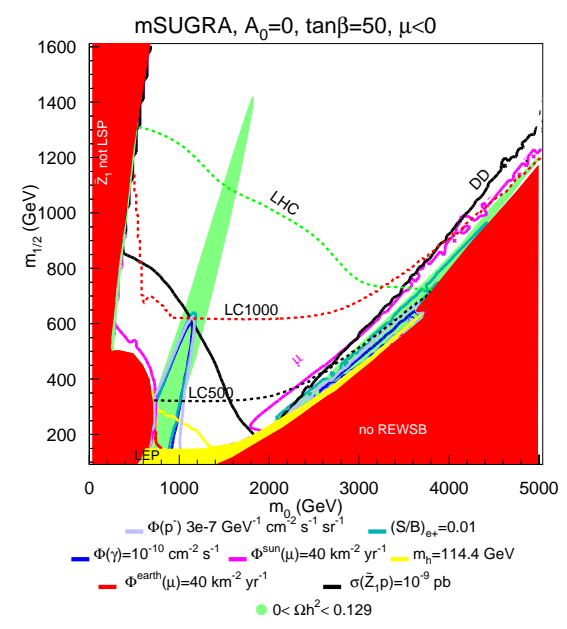

FIGURE 1. Expose of allowed regions of the mSUGRA model $m_{0} v s . m_{1 / 2}$ plane for $\tan \beta=50$, $A_{)}=0$ and $\mu<0$. We show dark matter allowed regions (green), collider reaches, and DD and IDD reach contours.

The three main regions of mSUGRA parameter space can be characterized by their direct and indirect detection rates: 1. For co-annihilation regions, one expects halo annihilation rates to be small, since co-annihilation cannot take place in the galactic halo. Also, DD and $v$-telescope rates may be very small. 2 . In the $A$-annihilation funnel, halo annihilation rates can be large, but DD and $v$-telescope rates can be small. 3 . In the FP region, all of halo annihilation, DD and $v$-telescope detection rates can be large.

\section{WELL-TEMPERED NEUTRALINOS IN SUGRA MODELS WITH NON-UNIVERSALITY}

While mSUGRA may be the most popular model for many SUSY analyses, there is strong motivation for SUGRA models with non-universality. A simple example ocurs in $S O(10)$ SUSY GUTs. Here, the Higgs multiplets may occupy the fundamental 10 of $S O(10)$, while matter scalars occupy the spinorial 16: one would expect in general $m_{10}^{2} \neq m_{16}^{2}$. With this single additional parameter, for any point in mSUGRA parameter space with too large $\Omega_{\widetilde{Z}_{1}} h^{2}$, one might dial[9] $m_{10}>m_{16}$ and reach mixed higgsino dark matter (even though one is not in the FP region), or one may dial $m_{10}^{2}$ to negative values and enter the $A$-funnel (even at low $\tan \beta$ ). The first of these situations is an example of a "well-tempered neutralino", wherein its composition is adjusted to gain the measured relic density[10]. When one gives it enough higgsino component to gain the measured relic density, one also increases the direct and indirect detection rates[11].

A variety of models with well-tempered neutralinos are shown in Fig. 2, There are one-parameter non-universal Higgs models $\left(N U H M_{\mu}\right.$ and $\left.N U H M_{A}\right)$ [9], models with mixed wino-bino-higgsino dark matter (MWDM1 and MWDM2)[12], high $M_{2}$ mixed bino-higsino dark matter (HM2DM)[13], low $M_{3}$ mixed bino-higgsino dark matter 


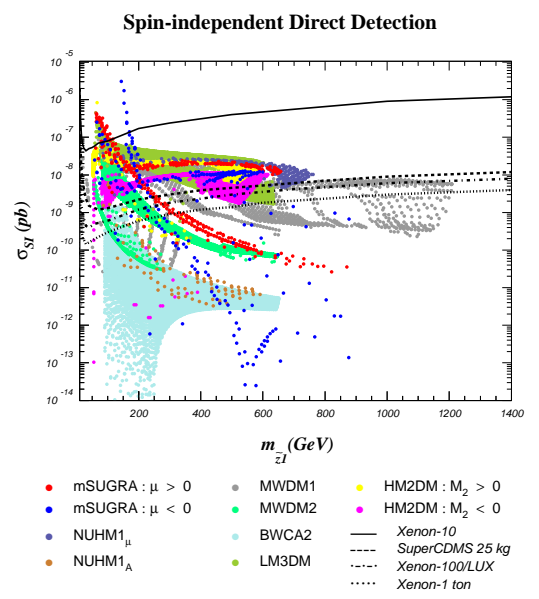

FIGURE 2. Direct detecion rates for a variety of models with well-tempered neutralinos. Note the asymptotic regions extending across $10^{-8} \mathrm{pb}$ !

(LM3DM) [14] and pure bino dark matter with its mass (not composition) tempered to allow for bino-wino co-annihilation (BWCA)[15]. Note, parameters are dialed so that every point has exactly the right relic density $\Omega_{\widetilde{Z}_{1}} h^{2} \simeq 0.11$. A few models with mass-tempering (such as BWCA) have low direct detection rates, but the models with neutralino composition tempering form an asymptote at $\sigma_{S I}\left(\widetilde{Z}_{1} p\right) \sim 10^{-8} \mathrm{pb}$. This cross section should be accessible to Xenon-100, LUX, WARP-140 and superCDMS. Thus, once the special $10^{-8} \mathrm{pb}$ SI scattering cross-section is well explored, either welltempered neutralino dark matter will be discovered, or this whole class of models will be excluded[11]! The FP region of mSUGRA of course falls in this region as well.

\section{MIXED AXION/AXINO DARK MATTER IN YUKAWA-UNIFIED MODELS}

One of the great successes of $S U(5)$ GUT theories was the prediction of $b-\tau$ Yukawa coupling unification. In the simplest $S O(10)$ SUSY GUT models, one expects the more restrictive condition of $t-b-\tau$ Yukawa coupling unification. It was recognized very early on that one criteria for this to occur in the MSSM is that $\tan \beta$ needs to be very large: $\tan \beta \sim 50$. At these high values of $\tan \beta$, SM-MSSM threshold corrections to the $b$-quark Yukawa coupling become very large[16]. These occur mainly through $\tilde{t}_{i} \widetilde{W}_{j}$ and $\tilde{g} \tilde{b}_{i}$ loop diagrams. The threshold corrections thus cause the entire calculation to depend on the spectrum of SUSY particles.

We assumed that the MSSM was the correct effective field theory between $M_{\text {weak }}$ and $M_{G U T}$, but that the parameter space at $M_{G U T}$ was that of $S O(10)$ :

$$
m_{16}, m_{10}, M_{D}^{2}, m_{1 / 2}, A_{0}, \tan \beta, \text { and } \operatorname{sign}(\mu) .
$$




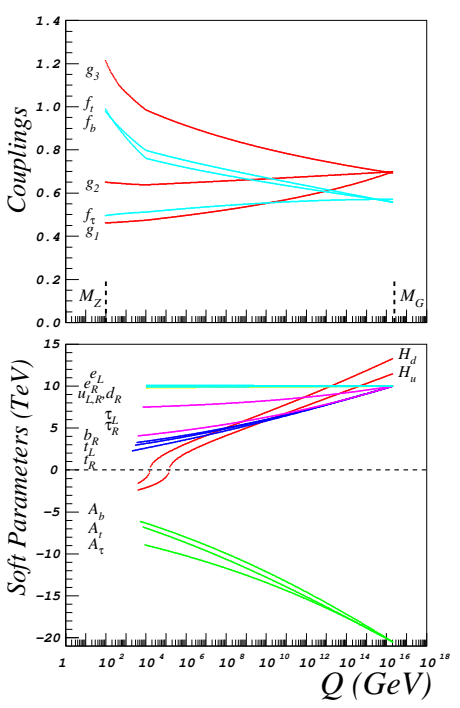

FIGURE 3. Evolution of gauge and Yukawa couplings (upper frame) and SSB terms (lower frame) in Yukawa-unified SUSY models.

Here $M_{D}$ parametrizes the splitting of the Higgs SSB terms and other scalars: $m_{H_{u, d}}^{2}=$ $m_{10}^{2} \mp 2 M_{D}^{2}$. Yukawa coupling unification succeeds best when the $D$-term splitting is only applied to the Higgs scalars, and not other matter scalars[17].

A scan over parameter space, using the Isajet/Isasugra spectrum generator (including full 2-loop RGE running and complete 1-loop sparticle mass and Yukawa threshold corrections) finds that Yukawa unified solutions can in fact be found. They are found for only very special choices of $S O(10)$ parameter choices: $1 . \tan \beta \sim 50,2 . m_{16} \sim 10$ $\mathrm{TeV}$, while $m_{1 / 2}$ is very small, $3 . A_{0}^{2}=2 m_{10}^{2}=4 m_{16}^{2}, 4 . \mu>0$, and 5. split Higgs mass at the GUT scale, with $m_{H_{u}}<m_{H_{d}}$. The latter criteria is need for an appropriate breakdown of EW symmetry. An example is given in Fig. 3.

The spectrum of SUSY particle generated for Yukawa unified SUSY is also very unique: 1. first/second generation matter scalars around $10 \mathrm{TeV}$, 2. third generation scalars around a few $\mathrm{TeV}$ due to the radiatively generated inverted scalar mass hierarchy[18], 3. gluino mass around $350-500 \mathrm{GeV}$ with charginos around $100-160$ $\mathrm{GeV}$ and $m_{\widetilde{Z}_{1}} \sim 50-80 \mathrm{GeV}[19]$.

Since $\mu$ and $m_{A}$ are typically a few TeV, the $\widetilde{Z}_{1}$ is nearly pure bino-like. The heavy scalars imply that the neutralino relic density $\Omega_{\widetilde{Z}_{1}} h^{2}$ is in the $10^{1}-10^{4}$ range: many orders of magnitude above its measured value. An elegant way to solve this Yukawaunified dark matter problem is to assume a Peccei-Quinn solution to the strong $C P$ problem, which then implies that a mixture of axions $a$ and axinos $\tilde{a}$ would actually constitute the dark matter. The $\widetilde{Z}_{1}$ lives a lifetime of about $1 \mathrm{sec}$, and decays before it can interfere with BBN: $\widetilde{Z}_{1} \rightarrow \gamma \tilde{a}$. From supergravity theory, we expect the gravitino 
mass $m_{3 / 2} \sim m_{16} \sim 10 \mathrm{TeV}$. This is actually very propitious, as it allows for a solution of the BBN/gravitino problem and allows for a reheat temperature of the universe $T_{R}$ in the range $10^{6}-10^{8} \mathrm{GeV}[20]$. This is not high enough for thermal leptogenesis, which requires $T_{R} \gtrsim 10^{10} \mathrm{GeV}$, but is high enough for non-thermal leptogenesis, wherein heavy right handed neutrino states are produced via inflaton decay, and which requires $T_{R} \gtrsim 10^{6}$ GeV.

With the above spectrum, we expect an assortment of rich signals from gluino pair production followed by 3-body gluino decays at the LHC[21]. It is also possible that an axion might be detected at direct axion search experiments. However, direct and indirect WIMP detection experiments should find a null result in the Yukawa-unified SUSY scenario with mixed axion/axino dark matter.

\section{ACKNOWLEDGMENTS}

I thank Dave Cline for organizing another fantastic Dark matter meeting. I also thanks my various collaborators listed in the references who did all the work.

\section{REFERENCES}

1. A. Chamseddine, R. Arnowitt and P. Nath, Phys. Rev. Lett. 49 (1982) 970; R. Barbieri, S. Ferrara and C. Savoy, Phys. Lett. B 119 (1982) 343; L. J. Hall, J. Lykken and S. Weinberg, Phys. Rev. D 27 (1983) 2359; for a review, see P. Nath, hep-ph/0307123.

2. H. Baer and X. Tata, Weak Scale Supersymmetry: From Superfields to Scattering Events, (Cambridge University Press, 2006).

3. D. N. Spergel et al. (WMAP Collaboration), Astrophys. J. Supp., 170 (2007) 377.

4. ISAJET, by H. Baer, F. Paige, S. Protopopescu and X. Tata, hep-ph/0312045.

5. H. Baer, C. Balázs, A. Belyaev, T. Krupovnickas and X. Tata, J. High Energy Phys. 0306 (2003) 054.

6. H. Baer, A. Belyaev, T. Krupovnickas and X. Tata, J. High Energy Phys. 0402 (2004) 007.

7. H. Baer, A. Belyaev, T. Krupovnickas and J. O' Farrill, JCAP0408 (2004) 005.

8. H. Baer and J. O'Farrill, JCAP 0404 (2004) 005.

9. H. Baer, A. Mustafayev, S. Profumo, A. Belyaev and X. Tata, Phys. Rev. D 71 (2005) 095008; H. Baer, A. Mustafayev, S. Profumo, A. Belyaev and X. Tata, J. High Energy Phys. 0507 (2005) 065.

10. N. Arkani-Hamed, A. Delgado and G. F. Giudice, Nucl. Phys. B 741 (2006) 108.

11. H. Baer, A. Mustafayev, E. K. Park and X. Tata, JCAP 0701 (2007) 017.

12. A. Birkedal-Hansen and B. Nelson, Phys. Rev. D 64 (2001) 015008; H. Baer, A. Mustafayev, E. K. Park and S. Profumo, J. High Energy Phys. 0507 (2005) 046.

13. H. Baer, A. Mustafayev, H. Summy and X. Tata, J. High Energy Phys. 0710 (2007) 088.

14. H. Baer, A. Mustafayev, E. K. Park, S. Profumo and X. Tata, J. High Energy Phys. 0604 (2006) 041.

15. H. Baer, T. Krupovnickas, A. Mustafayev, E. K. Park, S. Profumo and X. Tata, J. High Energy Phys. $0512(2005) 011$.

16. L. J. Hall, R. Rattazzi and U. Sarid, Phys. Rev. D 50 (1994) 7048.

17. T. Blazek, R. Dermisek and S. Raby, Phys. Rev. D 65 (2002) 115004; R. Dermisek, S. Raby, L. Roszkowski and R. Ruiz de Austri, J. High Energy Phys. 0304 (2003) 037; W. Altmannshofer, D. Guadagnoli, S. Raby and D. Straub, arXiv:0801.4363 (2008).

18. J. Bagger, J. Feng, N. Polonsky and R. Zhang, Phys. Lett. B 473 (2000) 264.

19. H. Baer and J. Ferrandis, Phys. Rev. Lett. 87 (2001) 211803; D. Auto, H. Baer, C. Balazs, A. Belyaev, J. Ferrandis and X. Tata, J. High Energy Phys. 0306 (2003) 023; H. Baer, S. Kraml, S. Sekmen and H. Summy, J. High Energy Phys. 0803 (2008) 056

20. H. Baer and H. Summy, Phys. Lett. B 666 (2008) 5.

21. H. Baer, S. Kraml, S. Sekmen and H. Summy, J. High Energy Phys. 0810 (2008) 079. 